\title{
APLICACIÓN DE UN PROGRAMA BASADO EN EL SOPORTE DE AUTONOMÍA EN LAS CLASES DE EDUCACIÓN FÍSICA
}

\section{IMPLEMENTATION OF AN AUTONOMY SUPPORT PROGRAM DURING PHYSICAL EDUCATION LESSONS}

Sánchez-Alcaraz, B.J. ${ }^{1}$, Álvarez-Ramiro, M. ${ }^{2}$, Manzano-Sánchez, D. ${ }^{3}$, GómezMármol, A. ${ }^{4}$, y Mayor-Gómez, M. $^{5}$

${ }^{1}$ Profesor Asociado. Facultad de Ciencias de la Actividad Física y del Deporte. Universidad de Murcia (España). Email: bjavier.sanchez@um.es.

2 Profesora Educación Física. I.E.S. Politécnico de Cartagena. (España). Email: miriama.ramiro@gmail.com.

3 Doctorando en Ciencias del Deporte. Facultad de Ciencias de la Actividad Física y del Deporte. Universidad de Murcia (España). Email: david.manzano@um.es.

4 Profesor Asociado. Facultad de Educación. Universidad de Murcia (España). Email: alberto.gomez1@um.es.

${ }^{5}$ Licenciada en Ciencias del Deporte. Universidad Católica de San Antonio (España). Email: mirimaygo@gmail.com

Código UNESCO: 5801 Teoría y Métodos Educativos

Clasificación Consejo de Europa: 5. Didáctica y Metodología

Recibido el 30 de enero de 2017

Aceptado el 28 de febrero de 2017

Correspondencia:

Sánchez-Alcaraz, B.J. bjavier.sanchez@um.es

\section{RESUMEN}

El objetivo de este estudio es conocer los efectos de un programa basado en el soporte de autonomía a través de las clases de Educación Física sobre las necesidades psicológicas básicas, la regulación conductual y la percepción del estilo interpersonal del docente. Los participantes del estudio fueron 61 alumnos ( 25 chicos y 36 chicas) con edades comprendidas entre los 14 y los 17 años de edad $(M=14.97$; $D T=.85)$, pertenecientes a dos Centros Educativos españoles. Los estudiantes fueron divididos aleatoriamente en dos grupos (control y experimental). Los instrumentos utilizados fueron la Escala de Satisfacción de Necesidades Psicológicas Básicas, el Cuestionario de la 
Regulación Conductual en el Deporte y el Cuestionario de Estilo interpersonal del docente. Los profesores aplicaron en el grupo experimental el programa basado en el soporte de autonomía durante las clases de Educación Física en un total de ocho semanas. Los resultados indicaron diferencias estadísticamente significativas respecto a la relación con los demás $(p<.01)$ y a la percepción del estilo autónomo del docente $(p<.05)$, no encontrándose diferencias significativas respecto al resto de variables. Como conclusión principal indicar que un programa basado en el fomento de la autonomía, puede ser óptimo para el incremento de las relaciones entre los alumnos.

PALABRAS CLAVE: Autonomía, Educación Física, Metodología, Motivación.

\section{ABSTRACT}

The purpose of this study is to know the effects of a autonomy support program through the Physical Education lessons on the psychological needs satisfaction, behavioral regulation and teacher interpersonal style. Sample were 61 students ( 25 boys and 36 girls) aged between 14 and 17 years $(M=14.97$; DT $=.85$ ), belonging to two educational centres in Spain. Students were randomly divided In two groups (control and experimental). The instruments used were the Basic Psychological Needs Satisfaction Scale, the Behavioral Regulation Questionnaire in Sport and the Interpersonal Teacher Style Questionnaire. The teachers applied in the experimental group the autonomy support program during the Physical Education lessons in a total of eight weeks. The results showed statistically significant differences regarding the relation with the others $(p<.01)$ and the perception of teacher autonomous style $(p<.05)$, not finding significant differences with respect to the other variables. As a main conclusion to indicate that a program based on the promotion of autonomy may be optimal for the increase relations between students.

KEYWORDS: Autonomy, Physical Education, Methodology, Motivation.

\section{INTRODUCCIÓN}

A día de hoy, es indudable que el sistema educativo español presenta unas tasas de rendimiento por parte de los alumnos muy inferiores a las esperadas, especialmente en secundaria ${ }^{1}$. Las tasas de abandono prematuro escolar en España doblan el promedio de la Unión Europea y triplican la estimación mundial, algo realmente preocupante ${ }^{2}$. Este hecho repercute en que no se beneficien de valores que son fundamentales para desenvolverse en la sociedad actual y que se incluyen implícitamente en la legislación actual, "Uno de los principios en los que se inspira el Sistema Educativo Español es la transmisión y puesta en práctica de valores que favorezcan la libertad personal, la responsabilidad, la ciudadanía democrática, la solidaridad, la tolerancia, la igualdad, el respeto y la justicia" ${ }^{3}$.

Es por ello por lo que se hace necesario ver, dentro de las escuelas, qué aspectos se deben tener en cuenta para que las clases se desarrollen de 
forma adecuada, tales como el clima motivacional ${ }^{4}$, necesidades psicológicas básicas como la autonomía ${ }^{5,6}$ y el papel fundamental del estilo docente y su metodología por su posible influencia en la experimentación de las emociones de los estudiantes ${ }^{7}$. Así, son numerosos los estudios en los que el soporte a la autonomía es una variable fundamental para el correcto funcionamiento de la enseñanza en general y para que los alumnos aprendan a desenvolverse en su contexto vital ${ }^{8}$. Por lo tanto, parece que un mayor soporte de autonomía por parte del profesor puede ayudar a evitar el fracaso escolar y mejorar el rendimiento académico ${ }^{9}, 10$. En este sentido, Oriol, et al. ${ }^{9}$ concluyen la necesidad de cambiar las metodologías de enseñanza para promover un aumento de la autonomía y motivación intrínseca, sobre todo teniendo en cuenta que la sociedad actual, hace que los estudiantes pierdan atención e interés rápidamente si están expuestos a actividades monótonas bajo metodologías tradicionales.

Este tipo de programas, por lo tanto, están basados en la teoría de la autodeterminación ${ }^{11}$. Esta teoría describe las condiciones bajo las cuales diversos factores sociales y contextuales promoverán diferentes tipos de motivación en las personas. Una de las bases en las que se apoya es en la subteoría de las necesidades psicológicas básicas ${ }^{11,12}$, donde se asume que existen tres necesidades básicas para el desarrollo y mantenimiento de la salud psicológica y/o bienestar personal: competencia (capacidad de realizar acciones con la seguridad de que el resultado sea aquel que se espera 0 desea), autonomía (capacidad de elegir aquella decisión que parezca más apropiada sin presiones externas) y relación con los demás (sentir que se puede contar con la colaboración y aceptación de las personas consideradas importantes). En la medida en la que estas necesidades psicológicas se satisfagan, se desarrollarán formas de motivación más o menos autodeterminadas (motivación intrínseca, motivación extrínseca y desmotivación). En concreto, según Moreno-Murcia, González-Cutre, Martín-Albo, y Cervelló ${ }^{13}$, es más probable que la persona alcance la motivación intrínseca (acción promovida por el disfrute obtenido durante la actividad) en aquellas situaciones en las que se permita la autonomía, se garantice la consecución de los objetivos establecidos (competencia) y se fomente la cohesión del grupo (relación con los demás). Sin embargo, si las necesidades básicas no son satisfechas, se incrementa la probabilidad de experimentar motivación extrínseca (acción promovida por un fin externo a la actividad) o incluso desmotivación (acción caracterizada por falta de interés hacia la práctica y por sentimientos de frustración), tal y como han demostrado Alvarez, Balaguer, Castillo, y Duda ${ }^{14}$ o Papaioannou, Tsigilis, Komidou, y Milosis ${ }^{15}$, siendo también fundamental considerar los contenidos que se imparten de cara a su alta influencia sobre todo en cuanto a la percepción de competencia ${ }^{16}$. En el caso de la motivación extrínseca, el fin que promueve la actividad puede ser a su vez provocado por considerar que la actividad física forma parte de un estilo de vida sano, activo y saludable (regulación integrada), por creer que la actividad es importante, pero no placentera (regulación identificada), por sentimientos de culpabilidad (regulación introyectada) y/o por presiones externas (regulación externa). 
De todo ello se desprende que existen una serie de mediadores que influyen en la motivación, sobre todo, la autonomía ${ }^{17}$. De ahí la importancia de centrarnos en la evaluación de los efectos producidos por un aumento de la autonomía de los estudiantes en su propio proceso de aprendizaje en lo que a motivación se refiere. Diversos trabajos han demostrado precisamente la intensa relación entre autonomía y motivación ${ }^{18,19}$. De esta forma, parece que la percepción de autonomía de los estudiantes necesita ser tenida en cuenta cuando hay implicados procesos psicológicos que intentan variar la motivación intrínseca ${ }^{17}$. Así, parece que un estilo del profesor basado en la cesión de autonomía con estrategias como la elección de las tareas por parte del alumno, podrían producir un aumento de la motivación intrínseca ${ }^{20,21}$ además de un mayor grado de satisfacción con la Educación Física si se logra un clima motivacional centrado en la tarea ${ }^{22,23 .}$

Por todo ello, el objetivo de este estudio es conocer los efectos de un programa basado en el soporte de autonomía a través de las clases de Educación Física sobre las necesidades psicológicas básicas, la regulación conductual mediada por la motivación y la percepción del estilo interpersonal del docente, hipotetizándose que la aplicación del programa en el grupo experimental, logrará unos mayores valores de motivación (especialmente intrínseca) y de satisfacción de las tres necesidades psicológicas básicas.

\section{MÉTODO}

\section{Participantes}

Los participantes del estudio fueron 61 alumnos (25 chicos y 36 chicas) con edades comprendidas entre los 14 y los 17 años de edad ( $M=14.97$; $D T=$ .85), pertenecientes a dos Centros Educativos de la Región de Murcia. La muestra se dividió aleatoriamente en dos grupos: control (32 estudiantes) y experimental (29 estudiantes).

\section{Procedimiento}

Selección de participantes y formación de los profesores

La selección de los Centros Educativos se realizó siguiendo los principios de proximidad y accesibilidad. Posteriormente, se desarrolló una reunión con los directores y profesores de Educación Física participantes donde se explicó la duración y desarrollo de la investigación. Se solicitaron los consentimientos informados de los padres y tutores de los alumnos participantes mientras que los profesores de Educación Física encargados de implantar el programa de Soporte de Autonomía recibieron un curso de formación de 10 horas distribuidas en 3 sesiones. El curso fue impartido por profesores especializados en esta metodología. Los contenidos del curso de formación fueron los siguientes: estructura de las clases de Educación Física basadas en el soporte de autonomía, herramientas pedagógicas para la adaptación de los contenidos de las clases a la metodología del soporte de autonomía y dossier de actividades para educar en autonomía a través del 
deporte. Una vez terminado el curso de formación, se realizó un test a los profesores para asegurar que los contenidos habían sido correctamente asimilados. Esta prueba estaba formada por 40 preguntas tipo test sobre los contenidos del curso de formación. Era necesario acertar un $80 \%$ de las preguntas para poder participar en el programa.

\section{Aplicación del programa de soporte de autonomía}

Una vez finalizado el curso de formación, se dividió la muestra aleatoriamente en dos grupos: control y experimental, y se realizó el pretest, consistente en la cumplimentación de los cuestionarios de satisfacción de las necesidades psicológicas básicas, motivación hacia la práctica deportiva, nivel de actividad física y percepción del alumno sobre el estilo interpersonal del profesor. Los alumnos completaron los cuestionarios de forma voluntaria y anónima, durante 25 minutos aproximadamente, en una clase de Educación Física, sin que ninguno de ellos presentara dudas a la hora de rellenarlos.

Posteriormente, los profesores aplicaron en el grupo experimental el programa basado en el soporte de autonomía durante las clases de Educación Física (dos clases de 60 minutos a la semana), en un total de ocho semanas. Los alumnos del grupo control realizaron sesiones de Educación Física con una metodología tradicional. Con el objeto de verificar que se estaba aplicando de forma correcta el programa de soporte de autonomía en el grupo experimental, todas las sesiones fueron grabadas y analizadas a través de una hoja de registro basada en las estrategias didácticas que dan soporte de autonomía al alumno. Además, durante la implantación del programa, se realizaron tres sesiones de seguimiento (duración total de 6 horas).

Los objetivos de estas sesiones eran: tratar las dificultades de los profesores durante la aplicación del programa, revisar los contenidos de las clases de Educación Física y ajustarlos a la metodología del soporte de autonomía y proporcionar diferentes feedbacks y estrategias para continuar con la implantación del programa. Además, se realizó un seguimiento y apoyo del proceso (vía telefónica, correo electrónico y página web) para resolver las posibles dudas que pudieran surgir $(24-26)$. Aunque esta verificación fue definida en términos de adherencia a los aspectos esenciales del programa, existía flexibilidad a la hora de su implementación a situaciones concretas del entorno del profesor. En este sentido, por ejemplo, cada profesor adaptó los ejercicios y tareas de sus Unidades Didácticas a la estructura y estrategias del soporte de autonomía, pero respetando los contenidos deportivos de su programación. El día posterior a la última sesión de la intervención basada en el soporte de autonomía se realizó el postest. El estudio tiene la aprobación del Comité de Bioética de la Universidad de Murcia. 


\section{Instrumentos}

Escala de Satisfacción de Necesidades Psicológicas Básicas. Se utilizó la validación al español (27) de la Psychological Need Satisfaction in Exercise Scale (PNSE) de Wilson, Rogers, Rodgers, y Wild (28). La PNSE utiliza 18 ítems, seis para evaluar cada una de las necesidades: competencia (e.g., "Tengo confianza para hacer los ejercicios más desafiantes"), autonomía (e.g., "Creo que puedo tomar decisiones en mis entrenamientos"), y relación con los demás (e.g., "Me siento unido a mis compañeros de entrenamiento porque ellos me aceptan como soy"). La sentencia previa fue "En mis entrenamientos..." y las respuestas fueron recogidas en una escala tipo Likert, cuyo rango de puntuación oscilaba entre 1 (Falso) y 6 (Verdadero). Los coeficientes de consistencia interna ( $\alpha$ de Cronbach) fueron para la autonomía: pre $=.81$, post $=.79$; para la competencia: pre $=.84$, post $=.81 ;$ para la relación con los demás: pre $=.71$, post $=.73$.

Cuestionario de la Regulación Conductual en el Deporte. Se utilizó la validación al español (Moreno-Murcia et al., 2011) del Behavioral Regulation in Sport Questionnaire (BRSQ) de Lonsdale, Hodge, y Rose (29). Este instrumento presenta 36 ítems que miden la regulación integrada (e.g., "Porque es parte de lo que soy"), identificada (e.g., "Porque los beneficios del deporte son importantes para mí"), introyectada (e.g., "Porque me sentiría avergonzado si lo abandono"), externa (e.g., "Porque otros me presionan a jugar"), la desmotivación (e.g., "Sin embargo, no sé por qué lo hago"), motivación intrínseca general (e.g., "Porque lo disfruto"), motivación intrínseca hacia el conocimiento (e.g., "Por el placer que me da el conocer más acerca de este deporte"), motivación intrínseca hacia la ejecución (e.g., "Porque disfruto cuando intento alcanzar metas a largo plazo") y motivación intrínseca hacia la estimulación (e.g., "Por el entusiasmo que siento cuando estoy implicado en la actividad"). Los ítems son evaluados por una escala Likert del 1 (nada verdadero) al 7 (muy verdadero). Los coeficientes de consistencia interna ( $\alpha$ de Cronbach) fueron para la motivación intrínseca general: pre $=.90$, post $=.90$; motivación intrínseca hacia el conocimiento: pre $=.90$, post $=.90$; motivación intrínseca de estimulación: pre $=.86$, post $=.86$; motivación intrínseca hacia la ejecución: pre $=.87$, post $=.87$; regulación integrada: pre $=.89$, post $=.89$; regulación identificada: pre $=.80$, post $=.80$; regulación introyectada: pre $=.76$, post $=.78$; regulación externa: pre $=.71$, post $=.67$; y desmotivación: pre $=.72$, post $=.73$.

Estilo interpersonal del docente: Se utilizó el cuestionario de Moreno-Murcia (En prensa) compuesto de 25 ítems que miden el estilo basado en el soporte de autonomía (e.g. "Valora nuestras ideas y sugerencias y nos deja proponer cosas") o el estilo controlador del docente (e.g. Habla continuamente y no permite que realicemos aportaciones en clase). Los ítems son evaluados en una escala tipo Likert de cinco posibilidades, desde 1 (seguro que no) a 5 (seguro que sí). Los coeficientes de consistencia interna ( $\alpha$ de Cronbach) fueron pre $=.84$ y post $=.74$ para el soporte de autonomía y pre $=.77$ y post $=$ .85 para el estilo controlador. 


\section{Análisis de datos}

En primer lugar se calcularon los estadísticos descripticos (media y desviación típica) de todas las variables objeto de estudio. Posteriormente, para calcular el efecto de la aplicación del programa basado en el soporte de autonomía y con el objetivo de que las variables contaminadoras, como el sexo y el curso de los estudiantes, tuviesen la menor influencia posible en los resultados de la investigación, se realizó una ponderación centrada mediante una regresión logística binaria. Después, se realizó una depuración de la muestra, eliminando los valores extremos, aplicando la distancia de mahalanobis ( $\alpha<.01$ considerada atípica) así como los valores de asimetría y curtosis ( $\alpha>3$ y $>10$ respectivamente) y se calculó una nueva variable a través de la prueba de ponderación centrada. Debido a que los datos no cumplían los criterios de normalidad, se realizaron pruebas no paramétricas, en concreto la prueba de Wilcoxon para conocer la influencia del tratamiento en cada uno de los grupos (control y experimental) y en cada una de las variables de estudio. El análisis de los datos se realizó utilizando el paquete estadístico SPSS 21.0 para Windows.

\section{RESULTADOS}

En primer lugar se realizó un análisis preliminar para comprobar si los grupos control y experimental presentaban diferencias significativas en los niveles de partida, no mostrando diferencias significativas en ninguna de las variables analizadas. Después, se comprobó los efectos del programa basado en el soporte de autonomía sobre la percepción del estilo interpersonal del docente, las necesidades psicológicas básicas y la regulación conductual, y cuyos resultados se muestran en la tabla 1.

Tabla 1. Efectos del programa basado en el soporte de autonomía sobre el nivel de actividad física, la percepción del estilo interpersonal del docente, las necesidades psicológicas básicas y la regulación conductual.

\begin{tabular}{|c|c|c|c|c|c|c|}
\hline & \multicolumn{3}{|c|}{ CONTROL } & \multicolumn{3}{|c|}{ EXPERIMENTAL } \\
\hline & $\begin{array}{l}\text { Pre-test } \\
M \pm D T\end{array}$ & $\begin{array}{c}\text { Post-test } \\
\text { M } \pm \text { DT }\end{array}$ & $p$ & $\begin{array}{l}\text { Pre-test } \\
M \pm D T\end{array}$ & $\begin{array}{l}\text { Post-test } \\
\text { M } \pm \text { DT }\end{array}$ & $p$ \\
\hline \multicolumn{7}{|l|}{ Estilo interpersonal del docente } \\
\hline Estilo controlador & $3.01 \pm .53$ & $3.08 \pm .64$ & .48 & $2.72 \pm .51$ & $2.70 \pm .73$ & .26 \\
\hline Estilo autónomo & $3.24 \pm .57$ & $3.31 \pm .67$ & .61 & $3.35 \pm .78$ & $3.75 \pm .78$ & $.02^{*}$ \\
\hline \multicolumn{7}{|l|}{ Necesidades psicológicas básicas } \\
\hline Autonomía & $3.65 \pm 1.09$ & $3.42 \pm 1.13$ & .26 & $3.08 \pm 1.15$ & $3.32 \pm .89$ & .27 \\
\hline Competencia & $4.36 \pm 1.15$ & $4.59 \pm 1.02$ & .27 & $4.75 \pm .90$ & $4.50 \pm .83$ & .19 \\
\hline Relación con los demás & $3.93 \pm 1.71$ & $4.20 \pm 1.03$ & .47 & $2.96 \pm .63$ & $4.58 \pm .77$ & $.00^{\star \star}$ \\
\hline \multicolumn{7}{|l|}{ Regulación conductual } \\
\hline Motivación general & $5.00 \pm 1.41$ & $5.57 \pm 1.85$ & .12 & $5.55 \pm 1.34$ & $5.59 \pm 1.12$ & .59 \\
\hline Motivación hacia el conocimiento & $4.89 \pm 1.65$ & $5.16 \pm 1.94$ & .43 & $5.43 \pm 1.48$ & $5.60 \pm 1.01$ & .57 \\
\hline Motivación hacia la estimulación & $4.86 \pm 1.53$ & $5.34 \pm 1.70$ & .20 & $5.31 \pm 1.13$ & $5.27 \pm 1.18$ & .86 \\
\hline Motivación hacia la consecución & $5.17 \pm 1.53$ & $5.42 \pm 1.68$ & .46 & $5.36 \pm 1.33$ & $5.48 \pm 1.03$ & .57 \\
\hline Regulación integrada & $5.08 \pm 1.55$ & $5.12 \pm 1.84$ & .90 & $4.95 \pm 1.31$ & $4.68 \pm 1.05$ & .26 \\
\hline Regulación identificada & $5.04 \pm 1.36$ & $5.22 \pm 1.64$ & .55 & $5.04 \pm 1.36$ & $4.83 \pm 1.18$ & .38 \\
\hline Regulación introyectada & $4.45 \pm 1.69$ & $3.87 \pm 1.86$ & .19 & $3.62 \pm 1.43$ & $3.54 \pm 1.57$ & .60 \\
\hline Regulación externa & $3.35 \pm 1.64$ & $2.60 \pm 1.20$ & $.03^{*}$ & $2.40 \pm 1.56$ & $2.75 \pm 1.47$ & .16 \\
\hline Desmotivación & $4.03 \pm 1.48$ & $2.82 \pm 1.42$ & $.00^{\star *}$ & $2.99 \pm 1.71$ & $1.95 \pm 1.45$ & .65 \\
\hline
\end{tabular}

Nota: $\mathrm{M}=$ Media; $\mathrm{DT}=$ Desviación típica; ${ }^{*} p<.05 ;{ }^{* \star} p<.01$. 
Tal y como se observa en la tabla 1, se muestran diferencias estadísticamente significativas para los que recibieron la intervención en cuanto al pre-test y al grupo control respecto a la relación con los demás $(p<.01)$ y en la percepción de un estilo autónomo por parte del docente $(p<.05)$. El grupo control en cambio, obtuvo una reducción estadísticamente significativa de los valores de regulación externa $(p<.05)$ y desmotivación $(p<.01)$, no encontrándose diferencias estadísticamente significativas en cuanto al resto de variables.

\section{DISCUSIÓN}

El objetivo de esta investigación fue conocer los efectos de un programa basado en el soporte de autonomía a través de las clases de Educación Física sobre las necesidades psicológicas básicas, la regulación conductual y la percepción del estilo interpersonal del docente. En este sentido, se ha comprobado como la aplicación del programa que cede autonomía a los alumnos mejoró la relación con los demás y la percepción de los alumnos de un estilo de enseñanza del profesor más autónomo. Estos resultados confirman los obtenidos por Moreno-Murcia, Gómez, y Cervelló ${ }^{6}$ donde indican que el tener la oportunidad de elegir o no entre las actividades programadas para la sesión o un clima motivacional orientado a la tarea, entre otras estrategias, incrementará la percepción de autonomía de los alumnos. Además, el programa basado en el soporte de autonomía mejoró las relaciones entre los alumnos, siguiendo la línea del trabajo de Moreno, Jiménez, Gil, Aspano, y Torrero ${ }^{30}$, que afirmaron que los alumnos a los que se les orienta hacia la tarea percibían unas mejores relaciones de cooperación y de ayuda con los compañeros.

Sin embargo, no parece que la aplicación del programa basado en el soporte de autonomía tenga efectos hacia la regulación conductual de los estudiantes, no encontrando entre los resultados aparentemente significativos respecto a ninguna de las variables motivacionales ni en cuanto a los valores de autonomía de los alumnos, contrariamente a los resultados expuestos en otros trabajos que han mostrado como un aumento en la autonomía en el alumnado incrementa los niveles de motivación autodeterminada y motivación intrínseca 13, 20, 21. En cuanto a la variable de percepción de competencia, tampoco se han obtenido cambios significativos, si bien esto puede deberse tal y como indican Julián et al. ${ }^{16}$ a que la influencia de los contenidos impartidos en clase, es un factor decisivo de cara al incremento de esta necesidad psicológica básica.

Como principales limitaciones de este trabajo, destacar en primer lugar el tamaño reducido de la muestra junto al reducido tiempo con el que se contó para la aplicación del modelo (ocho semanas). Futuros estudios deberían contemplar la posibilidad de ampliar la muestra de estudiantes y el tiempo de duración del tratamiento para comprobar los efectos del mismo e incluso poder aplicarlo en diferentes etapas educativas. Otra de las limitaciones del estudio, ha sido la propia de los contextos educativos, donde debido al entorno 
ecológico de intervención, donde se controla la cesión de autonomía por parte del profesor, pero esto no supone que el resto de estrategias que puede utilizar el profesor, y que no han sido controladas, puedan estar incidiendo en la percepción de los estudiantes del resto de variables ${ }^{13}$. Futuras investigaciones deberán tener en cuenta esta circunstancia (respecto al procedimiento) de cara a los resultados de las variables de regulación conductual. Además, sería de gran interés para futuras estudios que planten la posibilidad de aplicar modelos basados en el fomento de la autonomía y ver su incidencia en las variables contempladas en este estudio e incluso otras como el clima de aula, las conductas de los alumnos, el tiempo de compromiso motor entre otras.

En conclusión, y a la luz de los resultados obtenidos, parece que un programa basado en el soporte de autonomía durante el desarrollo de las clases de Educación Física puede servir para incrementar la satisfacción de la necesidad psicológica referida a la relación con los demás y la percepción de los alumnos de un estilo de enseñanza más autónomo del profesor.

\section{REFERENCIAS BIBLIOGRÁFICAS}

1. Ritacco, M. Amores, F. J. (2016). Perceptions of teachers and students of Initial Vocational Qualification Programs (PCPI). Development of the teaching-learning process in programs to prevent school failure in Secondary. Espiral. Cuadernos del profesorado, 9(18), 3-15.

2. Carrasco, S. Narciso, L. Bertrán, M. (2015). ¿Qué pueden hacer los centros públicos ante el abandono escolar prematuro? Explorando las medidas de apoyo al alumnado a través de dos estudios de caso en Cataluña en un contexto de crisis. Profesorado, revista de currículum y formación de profesorado, 16(3), 7692.

3. Ley Orgánica para la Mejora de la Calidad Educativa (LOMCE) (Ley Orgánica 8/2013, 9 de diciembre). Boletín Oficial del Estado, no 295, 2013, 10 diciembre.

4. Gutiérrez, M. López, E. y Ruiz, L. M. (2011). Clima motivacional en Educación Física: concordancia entre las percepciones de los alumnos y las de sus profesores. Revista de Psicología del Deporte, 20, 321-335.

5. Moreno-Murcia, J. Cervelló, E. García, T. Montero, C. Vera, J. (2012). Metas sociales, necesidades psicológicas básicas y motivación intrínseca como predictores de la percepción del esfuerzo en las clases de educación física. Revista de Psicología del Deporte, 21(2), 215-221.

6. Moreno-Murcia, J. A. Gómez, A. Cervelló, E. (2010). Un estudio del efecto de la cesión de autonomía en la motivación sobre las clases de educación física. Motricidad. European Journal of Human Movement, 24, 15-27.

7. López, S. Calderon, V. (2011). Gallup Student Poll: Measuring and Promoting, What is right with Students. En S. Donaldson, M. Csikzenthmihalyi, J. Nakamura, Applied positive psychology: Improving everyday life, schools, work, health, and society, (pp 117-134). New York: Routledge.

8. Escudero, J. M., Moreno-Murcia, J. A. (2012). Mejorar la educación, la autonomía de los centros y el servicio de inspección educativa. Avances en supervisión educativa: Revista de la Asociación de Inspectores de Educación de España, 17(1), 1-16.

9. Oriol, X. Amutio, A. Mendoza, M. Da Costa, S. Miranda, R. (2016). Emotional Creativity as predictor of intrinsic motivation and academic engagement in 
university students: The mediating role of positive emotions. Frontiers in Psychology, 7, 1-9.

10. Roth, G. Ron, T. Benita, M. (2009). Mothers' parenting practices and adolescents' learning from their mistakes in class: The mediating role of adolescent's selfdisclosure. Learning and Instruction, 19(6), 506-512.

11. Deci, E. L., Ryan, R. M. (1985). Intrinsic motivation and self-determination in human behaviour. New York: Plenum.

12. Deci, E. L., Ryan, R. M. (2000). The "what" and "why" of goal pursuits: Human needs and the self-determination of behaviour. Psychological Inquiry, 11, 227268.

13. Moreno-Murcia, J. A. González-Cutre, D. Martín-Albo, J. y Cervelló, E. (2010). Motivation and performance in physical education: an experimental test. Journal of Sports Science and Medicine, 9, 79-85.

14. Alvarez, M. S. Balaguer, I. Castillo, I. Duda, J. L. (2009). Coach autonomy support and quality of sport engagement in young soccer players. The Spanish Journal of Psychology, 12(1), 138-148.

15. Papaioannou, A. G. Tsigilis, N. Kosmidou, E. Milosis, D. (2007). Measuring perceived motivational climate in physical education. Journal of Teaching in Physical Education, 26, 236-259.

16. Julián, J. A. García L. Abarca, A. Zaragoza, J. (2014). Effect of gender and contents on physical activity and perceived competence in Physical Education. Revista Internacional de Ciencias del Deporte, 10 (36), 131-143.

17. Vallerand, R. J. Rousseau, F. L. (2001). Intrinsic and extrinsic motivation in sport and exercise. A review using the hierarchical model of intrinsic. En R. M. Singer, H. A. Hausenblas y C. M. Janelle (Eds.), Handbook of Sport Psychology (2nd ed., pp. 389-416). New York: Wiley.

18. Goudas, M. Biddle, S. Fox, K. Underwood, M. (1995). It ain't what you do, it's the way that you do it! Teaching style affects children's motivation in track and field lessons. The Sport Psychologist, 9, 254-264.

19. Vallerand, R. J. Perreault, S. (1999). Intrinsic and extrinsic motivation in sport: Toward ahierarchical model. En R. Lidor y M. Bar-Eli (Eds.), Sport psychology: Linking theory and practice (pp. 191-212). Morgatown, WV: Fitness Information Technology.

20. Prusak, K. Treasure, D. C. Darst, P. W. Pangrazzi, R. P. (2004). The effects of choice on the motivation of adolescent girls in physical education. Journal of Teaching in Physical Education, 23, 19-29.

21. Ward, J. (2005). The effects of choice on student motivation and physical activity behavior in physical education. Brigham: Brigham Young University.

22. Moreno-Murcia, J. A. Alonso, N. Martínez, C. Cervelló, E. (2005). Motivación, disciplina, coeducación y estado de flow en educación física: Diferencias según la satisfacción, la práctica deportiva y la frecuencia de práctica. Cuadernos de Psicología del Deporte, 5(1-2), 225-243.

23. Weigand, D. A. Burton, S. (2002). Manipulating achievement motivation in Physical Education by manipulating the motivational climate. European Journal of Sport Science, 2(1), 1-14.

24. Fullan, M. (2001). Leading in a Culture of Change. San Francisco (California): Jossey-Bass.

25. Little, M., Houston, D. (2003). Research into Practice through Professional Development. Remedial and Special Education, 24(2), 75-87.

26. Sinelnikov, O. (2009). Sport Education for Teachers: Professional Development when Introducing a Novel Curriculum Model. European Physical Education Review, 15, 91-114.

27. Moreno-Murcia, J. A. Marzo, J. C. Martínez, C. Conte, L. (2011). Validación de la 
Escala de "Satisfacción de las Necesidades Psicológicas Básicas" y del Cuestionario de la "Regulación Conductual en el Deporte" al contexto español. RICYDE, Revista Internacional de Ciencias del Deporte, 26(VII), 355-369.

28. Wilson, P. M. Rogers, W. T. Rodgers, W. M., Wild, T. C. (2006). The Psychological Need Satisfaction in Exercise Scale. Journal of Sport and Exercise Psychology, 28, 231- 251.

29. Lonsdale, C., Hodge, K., y Rose, E.A. (2008). The development of the Behavioral Regulation in Sport Questionnaire (BRSQ): Instrument development and initial validity evidence. Journal of Sport and Exercise Psychology, 30, 323-355.

30. Moreno, B. Jiménez, R. Gil., A. Aspano, M. I. Torreno, F. (2011). Análisis de la precepción del clima motivacional, necesidades psicológicas básicas, motivación autodeterminada y conductas de disciplina adolescentes en la clases de Educación Física. Motricidad. European Journal of Human Movement, 26, 1-24.

Referencias totales citadas: 30

Referencias citadas correspondientes a la Rev Ib CC Act Fis Dep: 0 